\section{Is screening for endometrial cancer necessary in asymptomatic women treated with tamoxifen for breast cancer?}

\section{U Chul Ju, Woo Dae Kang, Seok Mo Kim}

Department of Obstetrics and Gynecology, Chonnam National University Medical School, Gwangju, Korea (ddiamo94@gmail.com)

Objective: We investigated the endometrial pathologic results in women treated with tamoxifen for breast cancer and determined the appropriate diagnostic approach to oncologic endometrial pathologic change.

Methods: The medical records of 162 patients who underwent endometrial biopsy in patients treated with tamoxifen for breast cancer were retrospectively reviewed. We compared the clinical features between the patients with endometrial intraepithelial neoplasia or endometrial cancer (EIN/EC group) and the patients with other pathologic results (others group).

Results: EIN or EC were found in ten patients (6.2\%). $70.0 \%$ of EIN/EC group received tamoxifen treatment more than 3 years, while $71.7 \%$ of others group received less than 3 years $(p<0.001)$. Regardless of the presence of vaginal bleeding, EIN/EC was not found in patients with $\mathrm{ET}<5 \mathrm{~mm}$ by transvaginal ultrasonography. When ET was $\geq 5 \mathrm{~mm}$, the incidence rates of EIN/EC were only $2.9 \%$ in asymptomatic patients and $11.6 \%$ in symptomatic patients, respectively ( $\mathrm{p}=0.002)$. When ET was $\geq 8 \mathrm{~mm}$, the incidence rates of EIN/EC were $3.8 \%$ in asymptomatic patients and $12.0 \%$ in symptomatic patients, respectively $(\mathrm{p}=0.002)$. When ET was $<8$ $\mathrm{mm}, \mathrm{EIN} / \mathrm{EC}$ was not found in asymptomatic patients, while EIN/ EC was found in $7.1 \%$ of symptomatic patients.

Conclusion: The longer the use of tamoxifen, the greater the need for endometrial evaluation. However, unconditional endometrial evaluation should be avoided in patients without vaginal bleeding. When patients with vaginal bleeding show thick endometrium by TV-US, they need to undergo endometrial biopsy.

Poster (E11)

Conservative \& Fertility Preservation

https://doi.org/10.3802/jgo.2021.32.S1.E11

JGOG2051: The safety and efficacy of repeated high-dose luteal hormone (MPA) therapy for recurrent early-stage endometrial cancer patients

Kensuke Sakai, ' Wataru Yamagami, ${ }^{1, *}$ Nobuyuki Susumu, ${ }^{2}$ Yoshihito Yokoyama, ${ }^{3}$ Kazuhiro Takehara, ${ }^{4}$ Daisuke Aoki'

'Keio University School of Medicine, Tokyo, Japan (gami@z8.keio.jp) ${ }^{2}$ International University of Health and Welfare, Otawara, Japan

${ }^{3}$ Hirosaki University Graduate School of Medicine, Hirosaki, Japan

${ }^{4}$ National Hospital Organization Shikoku Cancer Center, Matsuyama, Japan
Background: The number of patients with endometrial cancer (EC) has been steadily increasing in Japan. Additionally, the number of adolescence and young adults with EC who desire fertility-sparing treatment has also increased. A previous metaanalysis showed that the initial rates of complete response (CR) to medroxyprogesterone acetate (MPA) therapy for EC and atypical endometrial hyperplasia (AEH) were $76 \%$ and $86 \%$, respectively; however, the recurrence rates were $26 \%$ and $41 \%$, respectively. Only some retrospective studies of a single institution on repeated MPA therapy were conducted for intrauterine recurrence following fertility-preserving therapy for EC or AEH, and showed favorable results. Therefore, multicenter prospective studies for repeated MPA therapy were highly demanded. Based on these backgrounds, the Japanese Gynecologic Oncology Group (JGOG) initiated this study to verify the efficacy and feasibility of repeated MPA therapy. Methods: JGOG conducts a multicenter phase II trial of repeated high-dose luteal hormone therapy for intrauterine recurrence following fertility-preserving therapy for $\mathrm{AEH}$ or EC. The major eligibility criteria are: 1) histologically diagnosed as AEH or G1, 2) myometrial invasion ruled out by magnetic resonance imaging, 3) extrauterine lesions ruled out by computed tomography, 4) 20-42 year old, and 5) strong desire and consent for fertility-sparing treatment. The major exclusion criteria are: 1 ) recurrence $\geq 3,2$ ) $\mathrm{BMI} \geq 40 \mathrm{~kg} / \mathrm{m}^{2}$ 3) previous medical history of thrombosis, 4) with multiple cancer with disease-free interval $<5$ years, and 5) with severe hepatic dysfunction. Patients are treated with oral MPA (600 mg/day) and are pathologically assessed by dilatation and curettage every 2 months until CR. Moreover, a hysterectomy will be recommended if the treatment duration exceeds 10 months. The primary end-point is 2-year recurrence-free survival during the follow-up period of 2 years. The secondary end-points are overall survival, progression-free survival, CR rate, pregnancy rate, adverse event, and pregnancy prognosis. One-hundred fifteen cases are needed to be recruited within 3 years, and currently Japanese 75 institutions are participating in this study. This study started in December 2020 in Japan, and the current plan is to collaborate with Korea and Taiwan.

\author{
Poster (E12) \\ Uterine Sarcoma \\ https://doi.org/10.3802/jgo.2021.32.S1.E12
}

\section{Preoperative clinical characteristics between uterine sarcoma and leiomyoma in patients with uterine mass: a case-control study}

Panlada Chantasartrassamee, Chompunoot Kongsawatvorakul, "Naparat Rermluk, 